\title{
¿Sobredosis de regulación? Algunos apuntes sobre la regulación farmacéutica*
}

Carolina Vivanco Vargas

Abogada por la Universidad de Lima. Miembro Asociado de ADV Editores - Revista ADVOCATUS.

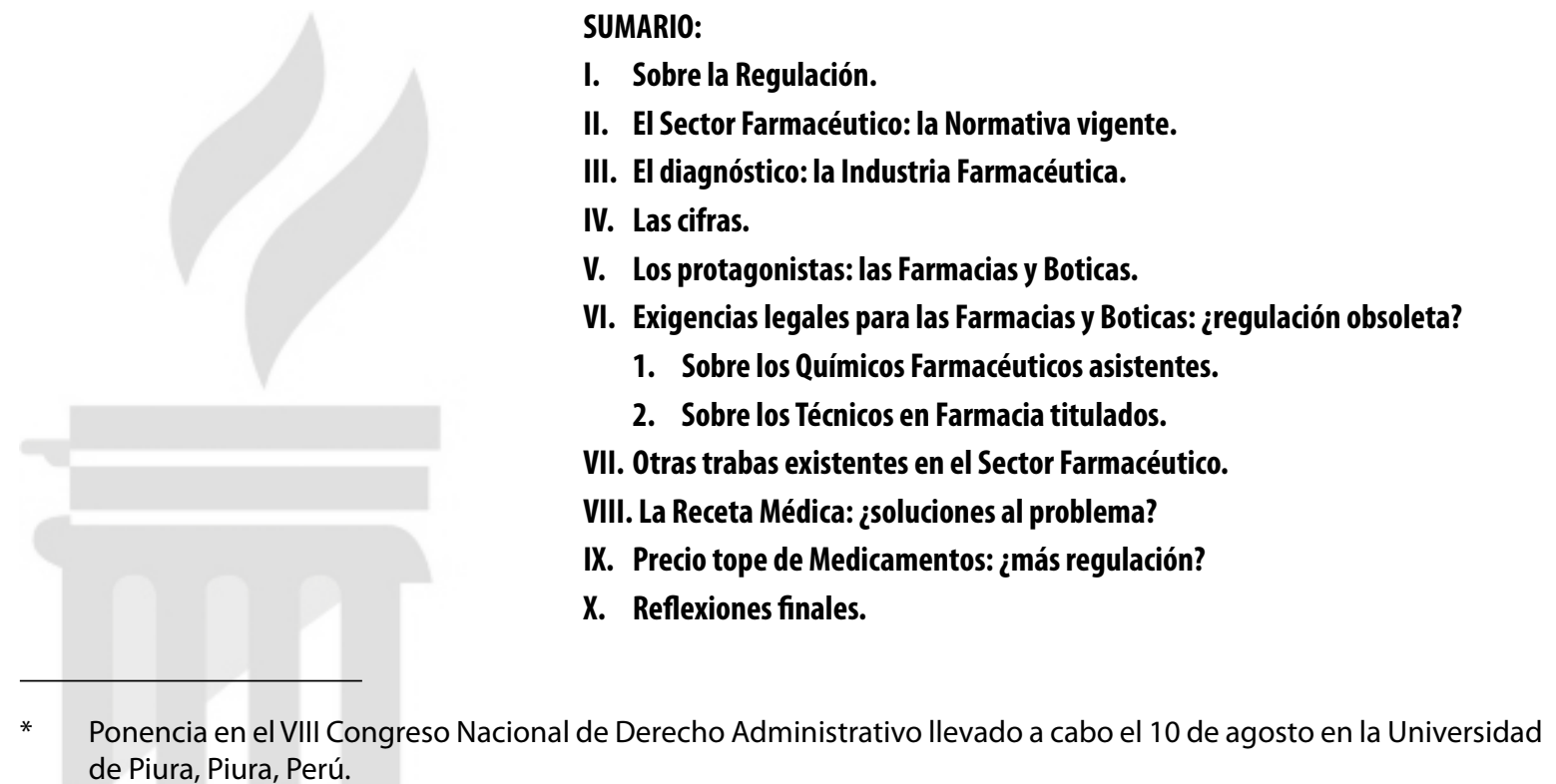




\title{
RESUMEN:
}

El presente artículo desarrolla la regulación farmacéutica desde el punto de vista de los títulos habilitantes necesarios para operar en dicha industria. Asimismo, analiza la situación actual del sector farmacéutico de acuerdo con los diversos estudios realizados. Asimismo, identifica algunas exigencias requeridas para el funcionamiento las farmacias y boticas que constituyen trabas para el adecuado desenvolvimiento de dichos establecimientos farmacéuticos, comentando así las resoluciones emitidas por INDECOPI sobre el particular.

Palabras Clave: Farmacéutico, Farmacias, Boticas, Regulación, Salud.

\begin{abstract}
:
The following article analyzes pharmaceutical regulations from the point of view of the qualifications that necessary to operate in said industry. It also analyzes the current situation of the pharmaceutical sector, according to various studies. Additionally, it identifies some prerequisites required for the operation of pharmacies and drugstores that constitute obstacles for the proper development of said pharmaceutical establishments, commenting thus on the resolutions issued by INDECOPI on the particular.
\end{abstract}

Keywords: Pharmacist, Pharmacies, Drugstores, Regulation, Health.

La industria farmacéutica es un mundo poco explorado desde el punto de vista legal el cual, por su importancia, merece ser tenido en consideración. En el presente artículo se analiza la regulación farmacéutica y los requisitos legales para el adecuado funcionamiento de las empresas involucradas en el sector, con el fin de reflexionar sobre si la normativa actual realmente regula de forma adecuada lo que, en la práctica sucede en el sector farmacéutico, o si se trata de normativa que, dado los cambios en la industria, resulta obsoleta o burocrática.

\section{SOBRE LA REGULACIÓN}

De acuerdo a la Constitución, en nuestro país existe la libertad de empresa. Esto quiere decir que los particulares se encuentran en plena libertad de poder desarrollar cualquier clase de actividad económica que se encuentre permitida por el ordenamiento jurídico, sin que deban existir limitaciones o exigencias que puedan menoscabar dicho derecho.

En efecto, la Constitución establece una serie de garantías y derechos que deben ser respetados durante la actividad diaria y comercial de las personas, naturales o jurídicas, que se desenvuelven en sociedad. El modelo que recoge es la econo- mía social de mercado, modelo que, como bien ha explicado nuestro Tribunal Constitucional:

“(...) parte de la premisa que el mejor sistema para la asignación y distribución de los recursos, es aquel que propicia la concertación libre entre oferta y demanda, puesto que de este modo se promueve el despliegue de las iniciativas de los seres humanos, se incentiva la competencia creadora y se impulsan las innovaciones tecnológicas. Al Estado en este esquema le corresponde crear las condiciones para que las actividades económicas privadas se desarrollen de manera libre y competitiva, procurándoles un marco para su desarrollo eficiente, que redunde en mejores productosy a precios competitivos para los consumidores yusuarios"11 (Subrayado agregado).

Ahora bien, la libre oferta y demanda se traduce en una serie de derechos reconocidos constitucionalmente, dentro de los cuales se encuentra el derecho a la libre empresa, recogido en el artículo $59^{\circ}$ de la Constitución, el cual señala que:

"El Estado estimula la creación de riqueza y garantiza la libertad de trabajo y la libertad de empresa, comercio e industria. El ejercicio de

1. Sentencia recaída en el Expediente $\mathrm{N}^{\circ}$ 0048-2004-Al. 
estas libertades no debe ser lesivo a la moral, nia la salud, nia la seguridad pública. El Estado brinda oportunidades de superación a los sectores que sufren cualquier desigualdad; en tal sentido, promueve las pequeñas empresas en todas sus modalidades"(Subrayado agregado).

De acuerdo con lo anterior, todos los ciudadanos tienen el derecho a efectuar actividades económicas en plena libertad. Sin embargo, se debe tener en cuenta que, dependiendo del tipo de actividad que se realice, existen ciertas reglas $y$ requisitos que deben ser acreditados. Ello, con el fin de salvaguardar los derechos e intereses de la colectividad con relación a la actividad económica que se pretenda realizar.

De esa manera es como nace el concepto de regulación, el cual se encuentra orientado a "armonizar la libre iniciativa con la defensa y promoción del bien común"2. Así, en palabras de Laguna, se entiende como regulación "el conjunto de actuaciones públicas a través de las que se ordena el ejercicio de la iniciativa privada, con la finalidad de proteger los derechos de los terceros, evitar la producción de daños y promover el interés general"3.

Precisamente, en ello consiste la potestad de policía administrativa, forma primaria de actuación de la Administración Pública para el mantenimiento del orden público y de protección y tutela de diversos bienes jurídicos relevantes para el bienestar general: dondequiera que existan cometidos públicos, su actuación deberá estar orientada a solventar y asumir roles de satisfacción de los intereses públicos. Concretamente, a través de la actividad de policía administrativa, el Estado, a través de sus distintos órganos, regula y restringe determinadas actividades de los particulares, exigiendo para su realización el cumplimiento de determinados requisitos o disposiciones, es decir, interviene, controla y ordena dichas actividades.
En otras palabras, la potestad de policía administrativa -que influye principalmente sobre los derechos de propiedad y libre empresa- permite que el Estado pueda ordenar la actividad de los particulares a través de reglamentaciones de derechos y planificación de la propiedad, así como a través del otorgamiento de títulos habilitantes para el desarrollo de sus actividadesfundamentalmente actividades económicas. Abarca, asimismo, el control de las actividades de los particulares a través de la inspección y fiscalización.

De acuerdo con lo antes mencionado, la regulación es la forma de manifestación que se utiliza para establecer los requisitos y autorizaciones que deben ser cumplidas a cabalidad por los actores del mercado. Más aun, para que la regulación sea efectiva y eficiente, debe recoger la realidad, estableciendo así requisitos y limitaciones que se adapten al propósito perseguido por la normativa y a tutelar el bien común.

\section{EL SECTOR FARMACÉUTICO: LA NORMATIVA VIGENTE}

Tal como se evidencia, a través de la regulación, el Estado establece una serie de límites o parámetros aplicables a las distintas actividades económicas que se desarrollan en el territorio.

Así, el sector farmacéutico no es ajeno a la regulación. Por el contrario, se trata de una industria que se encuentra minuciosamente regulada a través de la normativa emitida por los órganos del Ministerio de Salud —en adelante, "MIN$\mathrm{SA}^{\prime \prime}$ - que cuentan con las competencias para ello. Al respecto, la Dirección General de Dirección General de Medicamentos, Insumos y Drogas —en adelante, "DIGEMID" — es la entidad encargada de proponer las políticas en materia de productos farmacéuticos, dispositivos médicos y productos sanitarios, teniendo injerencia tanto en la regulación de los establecimientos

2. LAGUNA DE PAZ, José Carlos. "Derecho administrativo económico". Primera Edición. Navarra: Thomson Reuters, 2016, p. 27.

3. Ibid., p. 32. 
farmacéuticos, como en los productos en sí. Adicionalmente a ello, DIGEMID es la entidad que emite los títulos habilitantes necesarios para que las empresas del rubro puedan realizar sus actividades económicas y para autorizar la comercialización de los productos farmacéuticos, dispositivos médicos y productos sanitarios a través de los registros sanitarios.

Así, las actividades vinculadas a los productos farmacéuticos, tales como fabricación, importación, almacenamiento y comercialización, se encuentran reguladas, básicamente, en tres 3 normas: (i) Ley 29459, Ley de los productos farmacéuticos, dispositivos médicos y productos sanitarios -en adelante, "Ley de Productos Farmacéuticos"-; (ii) su Reglamento, aprobado por Decreto Supremo N ${ }^{\circ}$ 016-2011-SA —en adelante, "Reglamento de la Ley" - y (iii) el Decreto Supremo N014-2011-SA, Reglamento de Establecimientos Farmacéuticos -en adelante, "Reglamento de Establecimientos Farmacéuticos".

Adicionalmente a la normativa antes mencionada, la DIGEMID emite una serie de Directivas y el MINSA, por su parte, Resoluciones Ministeriales a fin de aclarar ciertos aspectos vinculados con el funcionamiento de los establecimientos farmacéuticos o sobre guías aplicables a los títulos habilitantes que deben ser obtenidos por los actores del sector.

Como se puede apreciar, la regulación vigente establece una serie de requisitos y títulos habilitantes, los cuales tienen por finalidad el salvaguardar la salud de los peruanos. Esto es, asegurando la más alta calidad de los productos farmacéuticos que se comercializan en nuestro territorio y supervisando el adecuado funcionamiento de los establecimientos farmacéuticos, en los cuales se almacena y comercializa los mencionados productos.

Ahora bien, la regulación, a grandes rasgos, divide las obligaciones teniendo en cuenta si se trata de la persona jurídica que realiza la actividad dentro del rubro o del producto en sí que se pretende comercializar, de acuerdo a las siguientes consideraciones: (i) Autorización a la persona jurídica: para poder efectuar cualquier tipo de actividad vinculada con los productos farmacéuticos, tales como, fabricación, importación, almacenamiento o comercialización, la persona jurídica deberá encontrarse constituida como uno de las clases de establecimientos farmacéuticos.

De acuerdo con el Reglamento de Establecimientos Farmacéuticos los referidos establecimientos se clasifican en seis 6 categorías:

a) Oficinas Farmacéuticas - farmacias o boticas: dedicadas a la comercialización directa de productos farmacéuticos, dispositivos médicos y productos sanitarios directamente al usuario final.

b) Farmacias de los establecimientos de salud: establecimientos que se encuentran dentro de un establecimiento de salud; por ejemplo, clínicas u hospitales.

c) Botiquines: dedicados al expendio de ciertos productos restringidos establecidos por DIGEMID de forma directa al usuario.

d) Droguerías: establecimiento dedicado a importar, exportar, comercializar, almacenar o distribuir productos farmacéuticos, dispositivos médicos y productos sanitarios.

e) Almacenes especializados: es lugar que se ubica dentro de un establecimiento de salud dedicado al almacenamiento y distribución de productos farmacéuticos, dispositivos médicos y productos sanitarios.

f) Laboratorios: establecimientos dedicados a la fabricación, envasado, fraccionamiento, acondicionado, reacondicionado o exportación de productos farmacéuticos, dispositivos médicos o productos sanitarios.

Así, dependiendo de la actividad que desea realizarse se debe solicitar la Autorización Sanitaria como un establecimiento farmacéutico 
determinado. Así, por ejemplo, si lo que se desea es fabricar productos farmacéuticos, se debe solicitar Autorización como laboratorio. Si sólo se desea importar y comercializar, deberán constituirse como una droguería.

Para obtener la Autorización Sanitaria como establecimiento farmacéutico es necesario que se acrediten una serie de requisitos, los cuales pueden variar dependiendo del tipo de establecimiento que se desee operar.

Cada establecimiento tiene una serie de disposiciones específicas aplicables dependiendo del tipo de actividad que realice. Así, por ejemplo, las droguerías se encuentran prohibidas de comercializar los productos farmacéuticos de forma directa al público general.

Por otro lado, en el caso de farmacias y boticas, cada uno de los locales debe contar con Autorización Sanitaria emitida por DIGEMID. Es decir, cada farmacia que existe en nuestro país - así pertenezca a una cadena de farmacias- debe contar con Autorización Sanitaria.

(ii) Autorización al producto farmacéutico: como regla general, la Ley de Productos Farmacéuticos establece que todos los productos que ingresen o se produzcan en nuestro país, deberán contar con registro sanitario. Dicho registro es emitido por DIGEMID.

Para obtener el referido registro, es necesario que se acrediten una serie de requisitos, tales como que el fabricante cuente con Buenas Prácticas de Manufactura, que la empresa que los almacena cuente con Buenas Prácticas de Almacenamiento, entre otros destinados a asegurar la calidad del producto en todas las etapas de producción y comercialización de los mismos.

En líneas generales, el registro sanitario habilita a su titular a fabricar el producto, importarlo y comercializarlo. En tal sentido, los laboratorios - fabricantes - y droguerías -importadores - son los titulares de los registros sanitarios de los productos que se comercializan en nuestro país.

Teniendo en cuenta ello, si bien las farmacias y boticas almacenan y comercializan los productos farmacéuticos en sus establecimientos, la normativa establece que estos no deben ser los titulares de los registros sanitarios. Ello obedece a la lógica de que los importadores y fabricantes son quienes deben certificar los productos, y los comercializadores o distribuidores tienen la obligación y responsabilidad de verificar si son productos que expenden cuenten con registro sanitario vigente.

De esta manera, DIGEMID ha establecido un sistema en el cual se intenta asegurar que los productos que lleguen a las manos de los ciudadanos cuenten con registro sanitario, lo que garantiza la calidad y seguridad de los mismos.

Tal como se puede apreciar, el régimen legal de los productos farmacéuticos comprende una serie de normas que regulan tanto el funcionamiento de los establecimientos farmacéuticos como los productos que se comercializan.

Así, la DIGEMID tiene como finalidad establecer una serie de requisitos que se encuentran orientados a salvaguardar la calidad y el correcto manejo de los productos farmacéuticos, los cuales por su importancia e injerencia en la salud de la población deben ser cuidadosamente regulados por el estado.

\section{EL DIAGNÓSTICO: LA INDUSTRIA FARMACÉUTICA}

La industria farmacéutica juega un rol estratégico a nivel mundial. Según el ranking del 2016 de Forbes, seis empresas de la industria farmacéutica se encuentran dentro de las 100 más importantes del mundo. Así tenemos a Pfizer, Novartis, Roche, Sanofi, Merck y Glaxosmithkline ${ }^{4}$.

4. Tal como lo establece el Reporte Sectorial de la SNI N 9, 2016. Disponible en: <http://www.sni.org.pe/wp-content/ uploads/2017/01/Octubre-2016-Industria-de-productos-farmac\%C3\%A9uticos.pdf $>$. 
Es decir, se trata de una industria de suma importancia a nivel mundial, la cual con el pasar de los años ha cobrado mayor relevancia, logrando expandir la variedad de productos a ofrecer en el mercado, así como incentivar la investigación de nuevos fármacos con mayor efectividad y eficiencia para el tratamiento y prevención de enfermedades.

En el caso peruano, existen ciento noventa y seis empresas que elaboran productos farmacéuticos, las cuales la mayoría se encuentran ubicadas en Lima. Si bien es cierto, contamos con un número considerable de empresas dedicadas a la fabricación de medicamentos, es importante tener en cuenta que el Perú es un país principalmente importador de productos farmacéuticos.

En efecto, de acuerdo con el Reporte Sectorial emitió por el Instituto de Estudios Económicos y Sociales de la Sociedad Nacional de Industrias —en adelante, "SNI" — en el año 2016, "el monto de las importaciones supera ampliamente el de las exportaciones. En el 2015, el monto exportado alcanzó los US\$ 49 millones, mientras que las importaciones ascendieron a US\$ 871 millones".

Las cifras reveladas en el Reporte de la SNI evidencian que el mercado farmacéutico local no se da abasto suficiente para cubrir la demanda de productos farmacéuticos de la población peruana. Sea porque los productos que se requieren para el tratamiento de enfermedades no se fabrican en nuestro país o porque resulta económicamente más viable el importar los productos antes que fabricarlos en nuestro territorio.

Si bien existen diversos motivos o justificaciones para explicar la situación antes descrita, en mi opinión, la regulación del sector farmacéutico actualmente vigente es un factor que juega un papel importante en el desarrollo de dicho sector en nuestro país.

Así, la regulación contiene un sinfín de requisitos —-muchas veces burocráticos-que deben ser acreditados por los laboratorios de productos farmacéuticos los cuales, en muchas ocasiones, generan un desincentivo para la inversión en el sector. Ello pues, genera que sea económicamente más atractivo el efectuar importaciones de los productos antes de apostar por establecer un laboratorio peruano con el fin de fabricar los productos en nuestro territorio.

Se debe tener en cuenta que en el mercado farmacéutico mundial existen actores que han ganado terreno en el sector, como por ejemplo China, quien durante el 2015 fue el mayor exportador de productos farmacéuticos a nivel mundial. Así, es posible concluir que países con mayor capacidad de inversión se encuentran en capacidad de fabricar productos de buena calidad y a un menor precio, situación que afecta a los productores locales los cuales no solo deben competir con los actores del mercado peruano, sino también con empresas que importan productos a menor precio de países que cuentan con una avanzada tecnología y con menores trabas regulatorias que cumplir.

Dado que se trata de una industria que juega un papel sumamente importante en la sociedad peruana, los laboratorios que desarrollan actividades dentro de nuestro territorio - sean de capital nacional o extranjero- se han agremiado en la Asociación Nacional de Laboratorios Farmacéuticos-en adelante, "Alafarpe" - . Así, a través de dicho gremio las empresas pueden coordinar de forma conjunta con las entidades gubernamentales que regulan el desenvolvimiento de sus actividades. Asimismo, Alafarpe tiene como objetivo ser un canal para divulgar determinada información de los productos existentes en el mercado, así como sobre temas de interés general vinculados con la salud y el tratamiento de enfermedades.

\section{LAS CIFRAS}

De acuerdo con el Reporte emitido por la SNI y con la información publicada por el Instituto Nacional de Estadística e Informática -en adelante, "INEI"-, en nuestro país son los hogares quienes consumen la mayor cantidad de productos farmacéuticos, seguido por los establecimientos de salud pública y privada, tal como se observa en el siguiente gráfico: 


\section{Gráfico $\mathrm{N}^{0} 4$}

Actividades económicasdemandantes de productos farmacéuticos

(Distribución porcentual)

\begin{tabular}{|c|c|}
\hline Consumo en hogares & 62,4 \\
\hline Salud pública & 13,1 \\
\hline 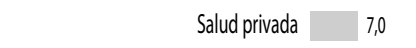 & \\
\hline Fabricación de productos farmacéuticos $\square$ 4,3 & \\
\hline Cría de animales $\quad 4,0$ & \\
\hline $\begin{array}{ll}\text { Administración pública y defensa } & 3,8\end{array}$ & \\
\hline Exportaciones 1,3 & \\
\hline Elaboración de bebidas no alcohólicas $\| 1,1$ & \\
\hline Resto de actividades $\quad 3,1$ & \\
\hline
\end{tabular}

Fuente: INEI

Elaboración: IEES - SNI

Tal como se puede apreciar, son los hogares, es decir, las personas de a pie, quienes consumen el $62.4 \%$ de la oferta de productos farmacéuticos en nuestro país.

Al respecto, es importante tener en cuenta que, de acuerdo con la normativa del sector, las personas naturales únicamente pueden adquirir productos farmacéuticos a través de dos - 2tipos de establecimientos: boticas o farmacias.

En efecto, la Ley de Productos Farmacéuticos establece que las farmacias y boticas son los canales autorizados de venta de productos farmacéuticos a personas naturales.

En tal sentido, el $62.4 \%$ de las ventas de medicamentos se efectúa a través de las farmacias y boticas existentes en nuestro país.

\section{LOS PROTAGONISTAS: LAS FARMACIAS Y BOTICAS}

Teniendo en cuenta las cifras antes desarrolladas, las farmacias y boticas juegan un papel fundamental en el mercado farmacéutico peruano.

En el caso de Lima, tradicionalmente se conocía a las farmacias y boticas como aquellos establecimientos independientes, que eran manejados por sus propietarios y se ubicaban cerca de las viviendas a fin que resulte accesible poder llegar a ellos. Con los años, ese concepto fue cambiando y se introdujo el concepto de las cadenas de farmacias y boticas.

En efecto, no es ninguna novedad el hecho que, desde hace unos años, las cadenas de farmacias y boticas vienen aumentando su presencia en nuestro país, con mayor incidencia en la capital. Así, uno puede encontrar farmacias o boticas de las cadenas de farmacias dentro de supermercados, centros comerciales y en las principales avenidas de Lima.

Se trata pues de un nuevo modelo de negocio que las farmacias y boticas vienen realizando: cadenas de establecimientos, con las mismas promociones, algunos productos exclusivos, sistemas de fidelización a los clientes, entre otros mecanismos de venta que hace unos años eran impensables.

Lamentablemente, la regulación farmacéutica no ha sabido adaptar los requisitos y exigencias impuestas para dichos establecimientos a la realidad actual, pues la normativa fue pensada y elaborada en base a las farmacias de "esquina" o las boticas "de barrio", tal como lo desarrollamos a continuación.

\section{EXIGENCIAS LEGALES PARA LAS FARMACIAS Y BOTICAS: ¿REGULACIÓN OBSOLETA?}

Como hemos mencionado en acápites anteriores, para que una farmacia o botica pueda funcionar bajo los parámetros de la regulación es indispensable que se encuentre autorizada por DIGEMID a través de una Autorización Sanitaria.

Para obtener dicho título habilitante, la normativa exige el cumplimiento de una serie de requisitos, los cuales se encuentran establecidos en el Reglamento de Establecimientos Farmacéuticos y en el Texto Único de Procedimientos Administrativos - en adelante, "TUPA de DIGEMID" -

Si bien, en mi opinión, me parece acertado que exista una regulación exhaustiva y minuciosa destinada a asegurar y salvaguardar la calidad de los productos farmacéuticos que se expenden a nivel nacional, existen ciertos requisitos - espe- 
cíficamente dos establecidos en el Reglamento de la Ley de Productos Farmacéuticos- que no se encuentran relacionados con ninguno de los objetivos planteados en la normativa sectorial, los cuales son los siguientes: (i) exigencia que para obtener la Autorización Sanitaria se deba contar con químicos farmacéuticos asistentes al Director Técnico ${ }^{5}$; y (ii) exigencia de que cada farmacia o botica cuente con un técnicos en farmacia titulados ${ }^{6}$.

\section{Sobre los químicos farmacéuticos asistentes.}

De acuerdo con la Ley de Productos Farmacéuticos y su Reglamento, las farmacias y boticas, como los demás establecimientos farmacéuticos, tienen la obligación de contar con la dirección técnica de un profesional químico farmacéutico. Dicho profesional es el responsable del adecuado funcionamiento del establecimiento con el fin de tutelar los requisitos de la calidad de los productos farmacéuticos que se expenden y comercializan, así como ejercer el control de calidad de los mismos y en la propia atención a los usuarios.

Así, la regulación establece que los establecimientos farmacéuticos deben contar con un único director técnico por cada establecimiento, el cual será responsable durante todo el horario de funcionamiento de la farmacia.
Es decir, la normativa exige la presencia permanente del Director Técnico durante todo el horario de funcionamiento del establecimiento farmacéutico.

Adicionalmente a ello, el Director Técnico de una farmacia no podrá desempeñarse como Director Técnico en otro establecimiento, es decir, existe exclusividad en la prestación del servicio:

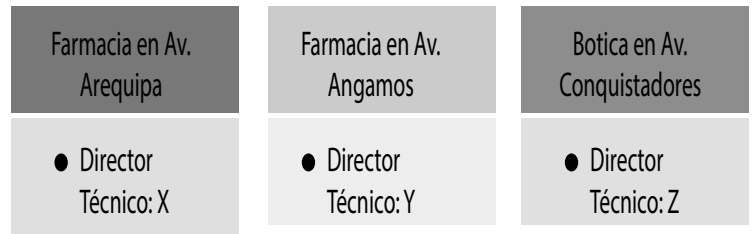

Ahora bien, como es predecible, existe la posibilidad que el Director Técnico se ausente en la realización de sus actividades - vacaciones, licencias, descanso médico, entre otras razones. Para ello, la regulación establece la posibilidad de contar con Químicos Farmacéuticos asistentes, con la finalidad que actúe de reemplazo ante la ausencia del Director Técnico.

No obstante lo señalado, el propio Reglamento de Establecimientos Farmacéuticos establece como un requisito necesario para obtener la Autorización Sanitaria como botica o farmacia la obligación de contar con químicos farmacéuticos asistentes de forma permanente.

5. “Artículo 18.- Requisitos para la Autorización Sanitaria de Funcionamiento.

El propietario o representante legal del establecimiento farmacéutico, para solicitar la Autorización Sanitaria de Funcionamiento, debe presentar los siguientes documentos, según se trate de:

A.- OFICINAS FARMACÉUTICAS: FARMACIAS O BOTICAS

(...)

- Nombres y números de colegiatura de los profesionales Químico Farmacéuticos asistentes;

- Horario de atención del establecimiento, del Director técnico y de los profesionales Químico Farmacéuticos asistentes.

e) Copia simple del certificado de habilidad profesional del Directortécnico y de los profesionales Químico Farmacéuticos asistentes (...)".

6. "Artículo 43.- Competencias del personal

El personal técnico en farmacia que labora en las farmacias y boticas debe contar con título que lo acredite como tal. El personal técnico en farmacia está impedido, bajo responsabilidad del Director técnico y del propietario del establecimiento, de realizar actos correspondientes a la dispensación de productos farmacéuticos de venta bajo receta médica o de ofrecer al usuario alternativas al medicamento prescrito". 
En una clara contravención a lo establecido en la Ley de Productos Farmacéuticos, el Reglamento establece un requisito ilegal a las boticas y farmacias, situación que vulnera el Principio de Legalidad y desnaturaliza la finalidad de la existencia de químicos asistentes, esto es, ser el reemplazo del Director Técnico, no un empleado adicional de las boticas y farmacias.

En efecto, si realmente la exigencia contenida en el Reglamento de la Ley de Productos Farmacéuticos - contar con químicos asistentes - fuera opcional el químico farmacéutico asistente sería personal que esporádicamente presta servicios a la farmacia - ante la ausencia del Director Técnico-, por lo que no sería posible establecer un horario de trabajo, dado que las ausencias del Director Técnico son impredecibles.

Sin embargo, DIGEMID exige como parte de los requisitos, para poder operar una botica o farmacia, la determinación de un horario de trabajo, situación que desnaturaliza la figura del químico asistente como personal volante y no permanente.

Adicionalmente a lo mencionado, no solo se trata del cumplimiento de un requisito para obtener la Autorización Sanitaria, sino que su incumplimiento genera la imposición de una infracción ${ }^{7}$.

En tal sentido, no solo se trata de una exigencia que restringe sin mayor sustento legal la expansión de este tipo de locales farmacéuticos, sino que también dificulta el adecuado desenvolvimiento de los mismos en el mercado, situación que se aparta de la finalidad de la regulación farmacéutica.

En efecto, se trata de una exigencia que carece de razonabilidad pues, si bien el interés público que se busca proteger es la salud pública, dicha necesitad se encuentra plenamente satisfecha con la exigencia de contar con un Químico
Farmacéutico registrado, el cual actúa de manera permanente como Director Técnico del establecimiento farmacéutico de acuerdo con lo dispuesto en la Ley de Productos Farmacéuticos. Así, el contar con químicos asistentes de forma permanente no genera una tutela más adecuada la salud de los usuarios, ni una mayor protección a los mismos.

Adicionalmente a ello, la DIGEMID y el MINSA no han tenido en consideración el sobrecosto que le genera a los titulares de los establecimientos farmacéuticos el tener como parte de su personal a un químico farmacéutico asistente -el cual solo puede ejercer sus funciones en caso de ausencia del Director Técnico- además de un Director Técnico por cada establecimiento.

Por otro lado, la regulación no tiene en cuenta el contexto actual de nuestro país pues, a la fecha se han creado las cadenas de farmacias y boticas, las cuales tienen una predominancia en diversas ciudades de nuestro país. Así, el imponer la exigencia de contar con un químico asistente, genera que por cada establecimiento se deban contratar a dos profesionales para realizar una misma labor, lo cual resulta absolutamente arbitrario e irracional.

Es importante tener en cuenta que las entidades de la Administración Pública deben establecer la regulación más idónea para tutelar el interés colectivo debiendo para ello establecer la menor cantidad de restricciones en las actividades económicas y cargas a los administrados. Sobre este punto, el propio Tribunal Constitucional en adelante, "TC" -; en la sentencia recaída en el Expediente $\mathrm{N}^{\circ}$ 007-2006-PD/TC a propósito de las restricciones a los horarios de funcionamiento de los establecimientos nocturnos establecidos por la municipalidad, concluyendo que:

"La restricción del horario de atención no constituye una medida idónea para la prosecución del objetivo que se propone la Munici-

7. Infracción tipificada en el numeral № 1 del Anexo N 01 del Reglamento de Establecimientos Farmacéuticos: "Por funcionar sin contar con director técnico o sin el personal exigido de acuerdo al Reglamento". 
palidad. En efecto, la protección de la integridad, la vida y la seguridad de los trabajadores de los establecimientos comerciales, así como de los concurrentes a ellos puede proveerse a través de la implementación de un adecuado servicio de la Policía Nacional y del servicio de Serenazgo de la propia Municipalidad e, incluso, establecerse como deber de los propios establecimientos comerciales, resultante de los servicios que brindan. En suma, la protección de aquellos derechos puede lograrse a través de un mayor y más adecuado servicio de seguridad, mas no a través de la restricción de los horarios de atención nocturnos y de madrugada" (subrayado agregado).

Siguiendo lo establecido por el TC en el caso concreto, el imponer como obligación el registro de un químico farmacéutico asistente por cada farmacia no resulta ser la medida más idónea para salvaguardar los derechos de los usuarios.

Así, es a través de la fiscalización posterior y de la implementación de mejores mecanismos de control que el MINSA y la DIGEMID pueden ejercer un mejor control del cumplimiento de las obligaciones establecidas en la normativa actual.

En efecto, no se trata de un problema en la regulación actual - Ley de Productos Farmacéuticos - sino en una adecuada fiscalización posterior, obligación atribuible en todo caso al MINSA y a la DIGEMID, la cual no puede ser trasladada a los administrados a través del establecimiento de prohibiciones, restricciones o exigencias que carecen de razonabilidad y que además son ilegales.

La presente problemática ha sido denunciada por la empresa Albis S.A. ante la Comisión de
Eliminación de Barreras Burocráticas -en adelante, "CEBB" - , del Instituto Nacional de Defensa de la Competencia y de la Protección de la Propiedad Intelectual-en adelante, "INDECOPI" - $y$, posteriormente, fue discutida en la Sala de Defensa de la Competencia del INDECOPI. En el presente caso, a través de la Resolución $N^{\circ}$ 0213-2018/SEL-INDECOPI, la Sala confirmó el criterio de la CEBB y declaró improcedente la denuncia presentada pues consideró que:

"(...) de la revisión conjunta de los artículos 18 y 41 del Decreto Supremo 014-2011-SA, Reglamento de Establecimientos Farmacéuticos, se advierte que la contratación de los químicos farmacéuticos asistentes resulta facultativa de las farmacias y/o boticas, no constituyendo una exigencia para el funcionamiento de las mismas".

En tal sentido, si bien la exigencia no ha sido declarada barrera burocrática lo cierto es que INDECOPI ha determinado que no es obligatorio contar con un asistente del Director Técnico. En tal sentido, el MINSA no podría -en teoríaexigir que las farmacias y boticas cuenten con un asistente del Director Técnico y tampoco podrían sancionar la inexistencia del mismo.

\section{Sobre los técnicos en farmacia titulados.}

A través del Reglamento de Establecimientos Farmacéuticos se estableció — de forma ilegal y arbitraria-que todo el personal técnico que laboran en las farmacias y boticas debía contar con título en dicha profesión ${ }^{8}$.

Sin embargo, dicha exigencia no encuentra sustento legal en ninguna de las normas que resultan aplicables. En efecto, la Ley 26842 —en adelante,

8. "Artículo 43.- Competencias del personal

El personal técnico en farmacia que labora en las farmacias y boticas debe contar con título que lo acredite como tal. El personal técnico en farmacia está impedido, bajo responsabilidad del Director técnico y del propietario del establecimiento, de realizar actos correspondientes a la dispensación de productos farmacéuticos de venta bajo receta médica o de ofrecer al usuario alternativas al medicamento prescrito.

EI Director técnico responde por la competencia técnica del o los profesionales Químicos-Farmacéuticos asistentes y del personal técnico" (Subrayado agregado). 
"Ley General de Salud"—, norma que establece los principios rectores aplicables a todos los ámbitos de la salud, no restringe la posibilidad de prestar servicios como técnico en farmacia a aquellos técnicos que hayan obtenido el título.

Por el contrario, la Ley General de Salud establece expresamente que existen tres (3) documentos que pueden ser utilizados para acreditar la culminación de los estudios técnicos, y, por ende, poder prestar servicios en el campo de la salud, en los siguientes términos:

"Quienes desarrollan actividades profesionales, técnicas o auxiliares relacionadas con la salud de las personas, se limitarán a ejercerlas en el área que el título, certificado o autorización legalmente expedidos determine" (subrayado agregado).

Es decir, la propia Ley General de Salud admite la posibilidad de que un técnico en farmacia preste sus servicios, siempre que acredite la culminación de los estudios técnicos en farmacia, hecho que puede ser acreditado utilizando tres clases de documentos, no restringiéndolo al título.

Así, la exigencia de contar con técnicos en farmacia titulados carece de sustento legal pues, la normativa aplicable es contraria a la restricción impuesta, dado que permite que los técnicos en farmacia que no sean titulados ejerzan sus actividades profesionales.

Coincidimos plenamente con el MINSA, en el extremo de que es sumamente importante que las personas que dispensan los medicamentos cuenten con los conocimientos necesarios para desempeñar adecuadamente su labor. Sin embargo, la única forma de demostrar dichas capacidades profesionales no es necesariamente el título, pues la propia Ley General de Salud establece la posibilidad de ejercer dichas actividades acreditando que se ha culminado con los estudios correspondientes — a través de un Certificado-. Es decir, el contar con un título no trae como consecuencia que el técnico en farmacia tenga un mayor o mejor conocimiento sobre el campo farmacéutico.

Lo antes mencionado resulta sumamente importante, pues se debe analizar siempre la finalidad de los requisitos que la normativa impone, puesto que los mismos deben responder a los objetivos que la norma persigue. En el caso concreto, el exigir el titulo para poder ejercer sus actividades como técnico en farmacia no acredita mayores condiciones para prestar sus servicios o pone en riesgo la salud de los usuarios.

Adicionalmente a ello, se debe tener presente el principio de jerarquía normativa, el cual implica el sometimiento de los poderes públicos a la Constitución y al resto de normas jurídicas, estableciendo un modo o rango de organizar las normas vigentes, consistente en hacer depender la validez de unas sobre otras.

En el caso concreto, respecto a las relaciones existentes entre la Ley y las normas de inferior jerarquía, la doctrina autorizada es clara y contundente al señalar que cualquier interpretación que se haga de un reglamento en nuestro sistema jurídico debe ser acorde al texto y principios de la ley que dicho reglamento desarrolla, puesto que, de lo contrario, dichas interpretaciones serían no solo ilegales, sino, lo que es mucho más grave, inconstitucionales:

"(...) la dualidad de las fuentes escritas se
ordena alrededor de un principio básico: la
absoluta prioridad de la Ley, expresión de
voluntad de la comunidad, respecto del Re-
glamento, expresión de voluntad subalterna
de la Administración. Esta prioridad es una
primacía puramente formal, por razón de la
fuente de legitimidad que diferencia a una ya
otranormay de la fuerza respectiva que de ello
extrae cada una, y se traduce en otro principio
de lajerarquíanormativa, que supone la abso-
luta subordinación del Reglamento de la Ley"

9. GARCÍA DE ENTERRÍA, Eduardo y RAMÓN FERNÁNDEZ, Tomás. "Curso de Derecho Administrativo". Tomo I. Lima: Palestra Editores, 2006, p. 254. 
(...) no hay ningún ámbito que pertenezca exclusivamente al Reglamento y que este pueda actuar al margen o prescindiendo (no ya contradiciendo, por supuesto) de la Ley (...)" (El subrayado es nuestro).

\section{REGISTRO NACIONAL DE FARMACIAS Y BOTICAS}

\begin{tabular}{|c|c|c|c|}
\hline Amazonas & 25 & 281 & 306 \\
\hline Ancash & 186 & 698 & 884 \\
\hline Apurímac & 31 & 140 & 171 \\
\hline Arequipa & 441 & 1798 & 2239 \\
\hline Ayacucho & 74 & 760 & 834 \\
\hline Cajamarca & 138 & 936 & 1074 \\
\hline Callao & 242 & 1679 & 1921 \\
\hline Cusco & 170 & 1068 & 1238 \\
\hline Huancavelica & 9 & 101 & 110 \\
\hline Huánuco & 12 & 488 & 500 \\
\hline Ica & 200 & 739 & 939 \\
\hline Junín & 228 & 1802 & 2030 \\
\hline La Libertad & 628 & 1534 & 2162 \\
\hline Lambayeque & 108 & 1259 & 1367 \\
\hline Lima & 81 & 812 & 893 \\
\hline Loreto & 29 & 321 & 350 \\
\hline Madre de Dios & 6 & 187 & 193 \\
\hline Moquegua & 24 & 107 & 131 \\
\hline Pasco & 11 & 280 & 291 \\
\hline Piura & 40 & 463 & 503 \\
\hline Puno & 95 & 698 & 793 \\
\hline San Martin & 42 & 679 & 721 \\
\hline Tacna & 29 & 457 & 486 \\
\hline Tumbes & 21 & 69 & 90 \\
\hline Ucayali & 18 & 431 & 449 \\
\hline
\end{tabular}

http://observatorio.digemid.minsa.gob.pe/PortalConsultas/ Consultas/ConsultaEstablecimientos.aspx?over=1

Así pues, tomando en consideración lo señalado, se puede advertir que a través del principio de jerarquía normativa se garantiza la validez de las normas jurídicas categóricamente inferiores respecto de las superiores, cuando la jerarqui- zación el único modo posible de organizar el poder normativo que tiene el Estado.

A través del Reglamento de Establecimientos Farmacéuticos, no es legalmente viable limitar el ejercicio de las actividades de un técnico en farmacia, tal como sucede en la norma comentada.

Sin perjuicio de la manifiesta ilegalidad de la disposición, es importante tener en cuenta que la regulación no toma en cuenta la situación actual de los técnicos en farmacia.

Así, a manera de ejemplo según la información publicada en la página de DIGEMID, a la fecha existen 17787 boticas y 2888 farmacias a nivel nacional:

Por otro lado, de acuerdo con la información proporcionada por la Dirección General de Educación Técnico-Productiva y Superior Tecnológica y Artística-DIGESUTPA, del Ministerio de Educación-MINEDU ${ }^{10}$ entre los años 2014 y 2016 egresaron veinte mil ciento quince -20 115- técnicos en farmacia a nivel nacional de los cuales sólo ocho mil setecientos cincuenta y dos - 8752 - se titularon, tal como se aprecia del siguiente cuadro:

Egresados y Titulados en Farmacia, 2014-2016

\begin{tabular}{|c|c|c|c|c|c|c|c|c|}
\hline Varia- \\
ble
\end{tabular}

Fuente: MINEDU- Censo Educativo, 2014-2016 Elaborado: MINEDU-DIGESUTPA

Es decir, de conformidad con la información oficial del MINEDU, sólo el $43.5 \%$ de los técnicos en farmacia se titula. 
Así, siguiendo lo establecido en el Reglamento de Establecimientos Farmacéuticos, sólo podrían desempeñarse como técnicos en farmacia A NIVEL NACIONAL 8752 egresados. Teniendo en cuenta que a nivel nacional existen veinte mil seiscientos setenta y cinco -20 675 - boticas y farmacias, es evidente que la cantidad de técnicos en farmacia titulados resulta insuficiente $-8752-$.

A través de la información antes expuesta se acredita que el requisito comentado no sólo es ilegal pues contraviene lo señalado en la Ley de Productos Farmacéuticos, sino que sí afecta el mercado farmacéutico de nuestro país, limitando de forma irracional y sin sustento técnico alguno la posibilidad que once mil trescientos sesenta y tres - 11 363 - técnicos en farmacia puedan desempeñarse como tal.

Adicionalmente a ello, restringe las posibilidades de empleo de miles de profesionales técnicos -11 363- que se ven impedidos que ejercer sus labores como consecuencia a una exigencia ilegal y carente de razonabilidad pues el contar con el título no genera una mayor preparación o conocimiento de la carreta técnica pues se trata de un requisito meramente administrativo.

En el presente caso, la empresa Albis S.A. denunció la cuestionada exigencia ante el INDECOPI, quién - acertadamente- declaró como barrera burocrática la obligación que los técnicos en farmacia deban contar con título para poder desempeñarse como tales en las farmacias y boticas. En efecto, el INDECOPI determinó que:

“(... el artículo 35 de la Ley 26842, Ley General de Salud, señala que quienes desarrollan actividades técnicas o auxiliares relacionadas con la salud de las personas (como el personal técnico en farmacia), se limitarán a ejercerlas en el área que el título, certificado o autorización legalmente expedidos determine. Es decir, el personal técnico en farmacia puede acreditar la realización de sus estudios no solo a través de un título profesional, sino que la indicada disposición de la Ley 26842, Ley General de Salud, habilita también la presentación de certificados expedidos por las instituciones educativas de educación superior, de conformidad lo provisto por la Ley 30512, Ley de Institutos y Escuelas de Educación Superior y de la Carrera Púbica de sus Docentes"11.

Tal como se puede apreciar, en los ambos casos comentados se evidencia un grave problema en la regulación actual: las normas no recogen la realidad actual del país del sector farmacéutico - específicamente con respecto a boticas y farmacias. En efecto, lejos de lograr que la normativa sea coherente con el funcionamiento de las empresas del sector, establece requisitos que se encuentran obsoletos y que resultan de muy difícil de poder ser cumplidos.

Es importante reflexionar sobre el problema en la regulación existente, pues podría afectar de forma directa a los actores de una industria que ostenta una gran relevancia en nuestra sociedad.

\section{OTRAS TRABAS EXISTENTES EN EL SECTOR FARMACÉUTICO}

Teniendo en cuenta la importancia que conlleva el mercado farmacéutico, en el año 2016, INDECOPI publicó las conclusiones obtenidas luego de haber efectuado una labor de análisis de las disposiciones legales vinculadas con el desenvolvimiento de los laboratorios en nuestro país. El documento fue elaborado con la colaboración de Alafarpe, quienes identificaron potenciales barreas burocráticas vinculadas con sus actividades.

Así, la Gerencia de Estudios Económicos del INDECOPI, como parte del Observatorio de disposiciones de la Administración Pública que afectarían a la Inversión Privada, publicó un informe en dónde identificó las principales

11. Resolución $\mathrm{N}^{\circ}$ 0214-2018/SEL-INDECOPI. 
disposiciones que podrían afectar la comercialización de productos farmacéuticos.

Al respecto, para la elaboración del informe, INDECOPI utilizó una metodología de dos etapas: (i) identificación de las potenciales barreras burocráticas, para lo cual Alafarpe reportó las disposiciones que podrían calificar como barrera; y (ii) medición del impacto económico de las potenciales barreras burocráticas a fin de estimar el costo o carga que representa para el empresario cumplir con los requisitos.

Tal como se puede apreciar, se trata de aspectos técnicos que afectan de forma frontal al desarrollo de la industria farmacéutica en nuestro país pues significan trabas para la fabricación, importación y comercialización de los productos farmacéuticos en nuestro territorio pues, de no contar con todos los requisitos requeridos por DIGEMID los productos no pueden ser comercializados, lo cual genera una afectación no solo a las empresas vinculadas al rubro sino a los consumidores que se verán afectados por escasez o por alza de precios.

Ahora bien, para poder entender a profundidad qué puede calificar como una"traba"a la inversión es necesario definir a las barreras burocráticas.

Al respecto, las barreras burocráticas son definidas como cualquier exigencia, requisito, limitación, prohibición y/o cobro dirigido a condicionar, restringir u obstaculizar el acceso y/o permanencia de los agentes económicos en el mercado y/o que puedan afectar a los administrados en la tramitación de procedimientos administrativos sujeto a las normas y/o principios que garantizan la simplificación administrativa.

El estudio elaborado por INDECOPI arrojó la existencia de diez potenciales barreras burocráticas vinculadas con el funcionamiento de los laboratorios en nuestro país. A manera de ejemplo, la exigencia que los insertos de los productos farmacéuticos no puedan ser aprobados por más de una agencia internacional; por ejemplo la Agencia Europea de Medicamentos -en adelante, "EMA" por sus siglas en inglés-, y la Agencia Administración de Alimentos y Drogas de los Estados Unidos de América —en adelante, "FDA" por sus siglas e inglés-, o denegarse a modificar el registro sanitario de productos ya registrados cuando se efectúe la ampliación de la fábrica extranjera en dónde son producidos-DIGEMID exige que se obtenga un nuevo registro sanitario y que el producto cambie de nombre-, entre otros ejemplos detallados por INDECOPI en el referido informe.

Como parte del estudio realizado, INDECOPI estimó que en el caso que una empresa deba cumplir con los diez requisitos establecidos como potenciales barreras burocráticas, esta tendrá que enfrentar un costo entre S/ 441034 y S/ 528 034 por cada producto que se desee importar.

Lo anterior evidencia de forma tangible y medible el impacto negativo que una regulación inadecuada puede causar en el mercado farmacéutico. Al margen si se tratan de requisitos legales o no, lo que se debe analizar es si realmente esas exigencias resultan necesarias y si se alinean a intentar obtener el objetivo detrás de las normas del sector: salvaguardar la salud de los administrados y ofrecer productos de calidad.

Así, a través de esta clase de estudios se puede evidenciar que, a la fecha, existe un grave problema en la regulación farmacéutica, el cual debe ser analizado a profundidad a fin de eliminar cualquier clase de traba que pueda afectar al adecuado desenvolvimiento de dichas empresas en el mercado, pues se trata de una industria sumamente sensible y necesaria para nuestra sociedad.

\section{LA RECETA MÉDICA: ¿SOLUCIONES AL PROBLEMA?}

La solución al problema planteado en la regulación es que la misma se adecúe a la realidad actual de las farmacias y boticas. Así, la normativa debe ser revisada en su integridad con el fin de detectar la evolución del mercado farmacéutico y cómo las normas que lo regulan se encuentran desfasadas, o si las disposiciones contenidas en ella cumplen adecuadamente los fines de la norma.

Esa es la solución que podría generar un verdadero cambio en la industria farmacéutica. 
Sin embargo, es poco realista pensar que ello podría suceder de forma adecuada y en un plazo reducido.

Por ello, el ordenamiento legal prevé un mecanismo específico que puede ser utilizado por los administrados, a fin de evitar la aplicación de exigencias que resulten ser ilegales o irracionales.

En efecto, a través de la Ley de Prevención y Eliminación de Barreras Burocráticas, aprobada por el Decreto Legislativo 1256 —en adelante, "Ley de Barreras" - se le otorga a los administrados, sean personas naturales o jurídicas que desarrollen actividades económicas, la posibilidad de denunciar ante la CEBB cualquier potencial barrera burocrática que pueda ser identificada y que le afecten en su desenvolvimiento económico.

Con relación al sector farmacéutico, el INDECOPI ha declarado barrera burocrática a una serie de requisitos establecidos en la normativa del sector.

A manera de ejemplo, a través de la Resolución Nº 0583-2017/CEB-INDECOPI, la CEBB declaró como barrera burocrática ilegal la exigencia de que el personal técnico que labora en las farmacias y boticas se encuentre titulado, materializado en el artículo $43^{\circ}$ del Reglamento de Establecimientos Farmacéuticos ${ }^{12}$.

Así, la CEBB concluyó acertadamente que dicha exigencia resulta ilegal, pues la Ley de Productos Farmacéuticos no establece requisitos o cualidades específicas para el personal técnico en farmacia que labora en las farmacias o boticas.

Por otro lado, a través de la Resolución $N^{\circ} 0523-$ 2017/CEB-INDECOPI, la CEBB declaró como barrera burocrática ilegal la prohibición de que una botica se encuentre ubicada al interior de una estación de servicio.

En el mencionado caso, Eckerd Perú S.A. denunció la prohibición de poder operar una botica dentro de una estación de servicio y el INDECOPI, acertadamente, concluyó que el MINSA no contaba con el sustento legal que faculte a establecer la referida prohibición.

Tal como se puede apreciar, el INDECOPI ha tenido a su cargo diversos casos vinculados con barreras burocráticas existentes en el sector farmacéutico, lo cual evidencia que determinados requisitos y exigencias establecidos en la regulación deben ser eliminados pues constituyen trabas para el adecuado desenvolvimiento del sector.

\section{PRECIO TOPE DE MEDICAMENTOS: ¿MÁS REGULACIÓN?}

Mucho se ha debatido sobre la regulación de precios en nuestro país. En efecto, se trata de un tema sumamente sensible en el cual existen posiciones totalmente contrapuestas sobre el tema.

En el caso concreto, el 1 de febrero de 2018 el congresista Javier Velásquez Quesquén presentó el Proyecto de Ley N 02371/2017-CR a través del cual propuso la regulación de precios máximos de los medicamentos básicos para enfermedades de mayor incidencia, gravedad y de alto costo.

Así, el Proyecto de Ley tiene por objetivo establecer una regulación que imponga precios máximos para la venta de determinados medicamentos considerados básicos. Ello, con la finalidad de asegurar el derecho constitucional a la salud.

De acuerdo con la exposición de motivos del Proyecto de Ley, el proponente sustenta que el resguardo adecuado del derecho a la salud comprende también el acceso a los medicamentos básicos y busca proteger el derecho de todos los peruanos al acceso a un tratamiento fijando precios considerados como "razonables".

Es decir, el Proyecto de Ley busca regular los precios de los medicamentos básicos estableciendo

12. La mencionada resolución ha sido apelada por el MINSA. Actualmente se encuentra siendo discutida en segunda instancia. 
precio tope pues así, de acuerdo con el Proyecto, se lograría resguardar el derecho a la salud.

Ahora bien, ¿cuáles son los medicamentos básicos?

El Proyecto de Ley los define como aquellos "de uso más frecuente en el tratamiento de las enfermedades de mayor incidencia, gravedad y de alto costo en nuestro país y que están considerados como tales por el Órgano Regulador de Precios Máximos de Medicamentos Básicos"13.

De la definición propuesta por el Proyecto no se logra desprender cuáles son los medicamentos que se encontrarían comprendidos en el ámbito de aplicación de la norma, pues le traslada la responsabilidad al Órgano Regulador de determinar dicho listado.

En efecto, a través del Proyecto de Ley, no solo se busca regular el precio tope de venta de medicamentos básicos, sino que crea un Órgano Regulador específico para que desempeñe dicha función.

El jefe del nuevo Órgano Regulador será un delegado designado por el Presidente de la República, quién actuará en su representación e integrado por el Ministro de Salud y el Ministro de Economía, de manera indelegable. Es decir, el Proyecto de Ley dispone que los propios ministros de estado sean quienes integren el Órgano Regulador.

La principal función del Órgano Regulador será el establecer, anualmente, el listado de medicamentos que sean considerados básicos, así como determinar el precio máximo de venta de los mismos. Dicho análisis, de acuerdo con el Proyecto de Ley, deberá realizarse bajo "(...) criterios objetivos y transparentes, metodología y oportunidad que se señale en el Reglamento de la presente Ley, que garanticen a la vez la investigación, desarrollo e innovación en la industria farmacéutica para promover un mercado competitivo"14.
Tal como se puede apreciar, el Proyecto de Ley busca regular los precios máximos de los medicamentos que se consideren como básicos, lo cual será de aplicación en todo el territorio de nuestro país, limitando así que los actores del mercado farmacéutico puedan comercializar dichos bienes a precios mayores a los que se fije.

Sin perjuicio que considero que legalmente no corresponde establecer precios tope - fijar precios - para el caso de los medicamentos, el Proyecto de Ley contiene una serie de vacíos o interrogantes que realmente resultan preocupantes.

Si bien es existe una gran preocupación por el acceso a los medicamentos en nuestro país, es importante no perder de vista que la regulación de precios es el último mecanismo que se debe de utilizar cuando el mercado funciona adecuadamente bajo la oferta y demanda.

Así, considero que en el caso concreto el Proyecto de Ley no solo contraviene una serie de principios de normas expresas, sino que no se encuentra debidamente sustentado.

En efecto, el Proyecto de Ley ni siquiera ha podido determinar los criterios y parámetros objetivos que se deberán aplicar a fin de determinar cuáles medicamentos deben ser considerados "básicos".

Ese es uno de los aspectos principales de cuestionamiento, pues es el punto de partida para poder determinar si realmente es necesario y las fallas del mercado justifican intervención en el precio. Caso contrario, ¿cómo se puede detectar la existencia de un problema en los precios cuando no se sabe ni siquiera cuáles medicamentos se encuentran comprendidos?

El Proyecto de Ley es muy escueto pues no analiza los aspectos más importantes que deben ser desarrollados en él, como lo es la determinación

13. Artículo 2 del Proyecto de Ley.

14. Artículo 3 del Proyecto de Ley. 
de los medicamentos que se encuentran comprendidos.

En esa línea, no determina ni un solo criterio que deba ser tomado en cuenta por el Órgano Regulador para establecer el listado de medicamentos básicos. Es decir, se trata de un Proyecto de Ley que carece de un contenido real, pues sus disposiciones son tan generales que ni siquiera establece un criterio lógico para poder determinar qué medicamentos se encuentran contemplados en él.

De esa misma forma, no ha establecido la metodología que deberá seguirse a fin de establecer el precio tope que se deberá cobrar por cada uno de los medicamentos. En efecto, no señala pasos o procedimientos mínimos que deberán ser realizados con el fin de garantizar una adecuada determinación del precio tope.

A manera de ejemplo, consideramos que se deberán de efectuar una serie de estudios de mercado, económicos, analizando el costo del producto y el precio de venta, la disponibilidad de genéricos en el mercado, entre otros factores que son garantías mínimas que los actores del mercado farmacéutico que se verán afectados con la medida — droguerías, laboratorios, farmacias- deberían conocer pues sus negocios se verán afectados de manera significativa.

Finalmente, el Proyecto de Ley no establece cuáles actores del sector farmacéutico se deberán acatar a esta medida. Al respecto, cabe señalar que la industria farmacéutica se compone por una serie de empresas que juegan un rol específico en ella: los laboratorios - fabricantes-; las droguerías -importan y comercializan-; las farmacias y boticas - puntos de venta-; farmacias de establecimientos de salud -clínicas, hospitales-y establecimientos comerciales - productos farmacéuticos de bajo riesgo sanitario-.
De acuerdo con lo anterior nos preguntamos, ¿las farmacias de los establecimientos de salud -clínicas, hospitales- deberán acatar el precio máximo? ¿Se trata de una medida que únicamente afectará a los puntos de venta farmacias, boticas-?

Son interrogantes que, de la lectura del Proyecto de Ley, no pueden ser absueltas.

\section{REFLEXIONES FINALES}

La industria farmacéutica es uno de los sectores que juega un rol protagónico en nuestra sociedad, pues se encuentra íntimamente relacionado con la provisión de aquellos insumos que resultan indispensables para el tratamiento de las diversas enfermedades que aquejan a la población.

Al tratarse de una industria tan sensible la regulación para el desarrollo de sus actividades económicas, es bastante exhaustiva y protectora de los usuarios finales que recibirán los productos. Ello, con el objetivo de garantizar la mejor calidad de los productos que serán puestos a disposición dentro del mercado.

Si bien existen determinadas exigencias que resultan coherentes y que persiguen la finalidad de la normativa, se pueden detectar una serie de exigencias que, lejos de salvaguardar la calidad de los productos farmacéuticos, imponen requisitos adicionales que traen como consecuencias ineficiencias en el desarrollo de las actividades económicas de las empresas del sector.

En mi opinión, se debe de analizar nuevamente la regulación ahora vigente con el fin de detectar los requisitos que resulten obsoletos, pues la realidad de la industria ha ido cambiando con los años y resulta necesario un ajuste al contenido de las normas, con el fin de eliminar aquellos requisitos que no se encuentren alineados a la finalidad de la normativa. 